\title{
The Source Material for Large Solar Energetic Particle Events
}

\author{
R.A. Mewaldt and C.M.S. Cohen \\ Department of Physics, California Institute of Technology, Pasadena, California \\ G.M. Mason \\ Applied Physics Laboratory/Johns Hopkins University, Laurel MD
}

\begin{abstract}
We review evidence regarding the origin of material accelerated in large, gradual solar energetic particle (SEP) events. According to the two-class paradigm in place at the start of solar cycle 23, impulsive SEP events accelerate heated flare material, while gradual SEP events are accelerated out of the solar wind by shocks driven by fast coronal mass ejections (CMEs). However, new data from solar cycle 23 has shown that the energetic ions in gradual events often include composition signatures associated with impulsive events, including enrichments in ${ }^{3} \mathrm{He}$, heavy elements such as Fe, and ionic charge states indicative of $\sim 10 \mathrm{MK}$ temperatures. In addition, gradual SEP events differ in composition from bulk solar wind in several key respects, implying that solar wind is not the principal seed population for these events. Several lines of evidence show that CME-driven shocks accelerate principally suprathermal ions with velocities several times that of the solar wind. The suprathermal pool incorporates ions from impulsive solar flares and previous gradual events, CIR events, pickup ions, CME ejecta, and the suprathermal tail of the solar wind. This paper reviews evidence for the sources of ions accelerated in gradual SEP events, considers the composition and available densities of suprathermal ions, and describes models that attempt to account for the surprisingly variable composition of gradual SEP events. We find that below $\sim 1 \mathrm{MeV} /$ nucleon almost all events are Fe-rich compared to the average 5 to $12 \mathrm{MeV} /$ nucleon SEP composition. Iron-rich SEP events above $10 \mathrm{MeV} /$ nucleon occur mainly during periods when the intensity of suprathermal iron in the inner heliosphere is high, due mainly to previous gradual events.
\end{abstract}

\section{INTRODUCTION}

According to the paradigm that was established during the late 1980's and early 1990's there are two classes of solar energetic particle (SEP) events (Reames, 1995a). "Impulsive" events are generally small events associated with impulsive $\mathrm{x}$-ray flares that are enriched in ${ }^{3} \mathrm{He}$ and

Solar Eruptions and Energetic Particles

Geophysical Monograph Series 165

Copyright 2006 by the American Geophysical Union

10.1029/165GM12 heavy elements such as Fe. Measurements at $\sim 1 \mathrm{MeV} /$ nucleon showed that the ions in impulsive events had average charge states characteristic of temperatures of $\sim 5$ to $10 \mathrm{MK}$. "Gradual" events on the other hand, are accelerated by CME-driven shocks, with abundances similar to those in the corona, and charge states characteristic of $\sim 2-3 \mathrm{MK}$. It was generally assumed that impulsive events accelerate flareheated material, while gradual events accelerate coronal material and the ambient solar wind.

During the past solar maximum it became possible to measure the composition of SEP events accurately over a very broad energy interval that extended down to suprathermal 
energies ( $40 \mathrm{keV} /$ nucleon). These data were supplemented by isotopic and ionic charge-state data of unprecedented accuracy. These new observations showed that gradual events are accelerated from additional seed populations than just solar wind.

In order to understand the energy scales involved, it is useful to refer to Figure 1, which shows the measured fluence of oxygen from solar wind to galactic cosmic-ray energies from September 1997 to June 2000. Also shown are the contributions that various examples of solar and interplanetary events made to this 33-month spectrum. Much of the progress in understanding the source material of SEP events has come from the comparison of composition studies over a broad energy interval.

\subsection{New Observations of Impulsive and Gradual Events}

Data from this last solar maximum have shown that solar particle events are more complex than indicated in the picture described above [see also von Rosenvinge and Cane (2005)].

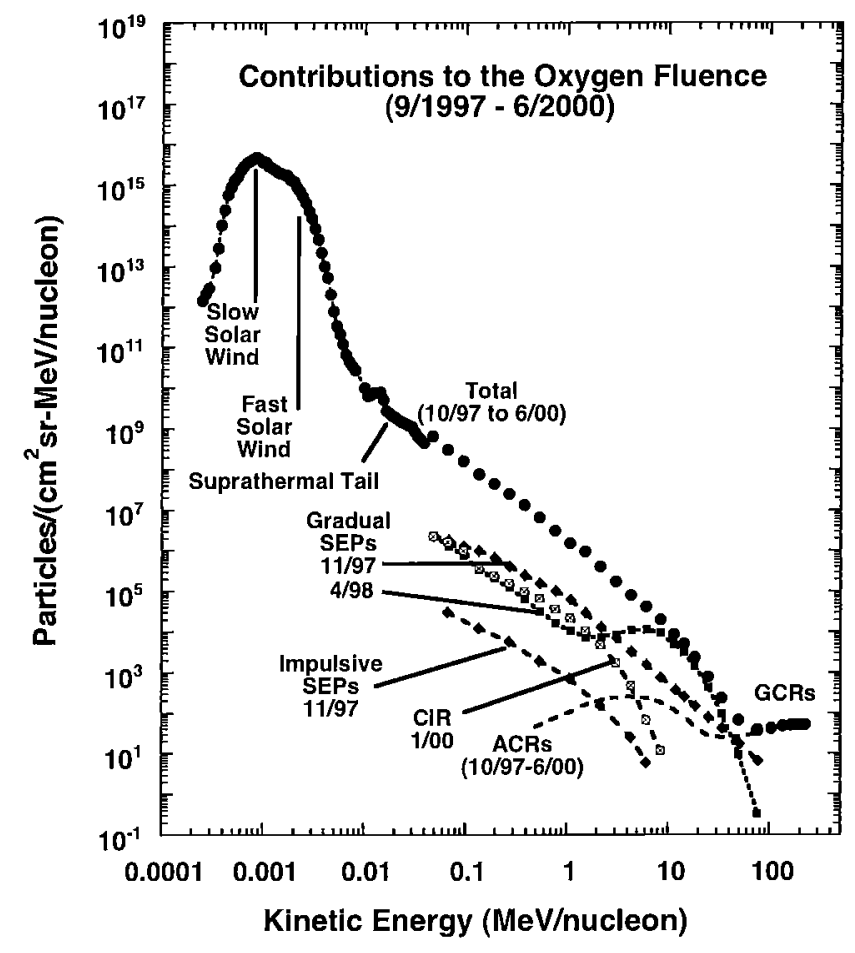

Figure 1. Fluence of energetic oxygen nuclei from solar wind to galactic cosmic ray energies. The fluence spectra $>40 \mathrm{keV} /$ nucleon from the ULEIS, SIS, and CRIS instruments on ACE were accumulated from September 1997 through June, 2000. Fluences $<0.04 \mathrm{MeV} /$ nucleon from the SWICS instrument were accumulated from January through December 1999 and multiplied by a factor of $11 / 4$. Also shown are the contributions to the overall fluence by various solar and interplanetary events (from Mewaldt and Mason, 2005)
In particular, with new instruments that could resolve ${ }^{3} \mathrm{He}$ in much lower concentrations $\left({ }^{3} \mathrm{He} /{ }^{4} \mathrm{He}\right.$ from $\sim 0.001$ to 0.1 ), it was found that in the energy range from $\sim 0.1$ to $\sim 30 \mathrm{MeV} /$ nucleon most gradual events have ${ }^{3} \mathrm{He} /{ }^{4} \mathrm{He}$ ratios well above that of the solar-wind ratio of $4 \times 10^{-4}$ (Cohen et al., 1999, Mason et al., 1999a, Wiedenbeck et al., 2000, Torsti, Laivola, and Kocharov, 2003).

In addition, Cohen et al. (1999) found that a large fraction of gradual events are enriched in Fe and other heavy elements at energies $>10 \mathrm{MeV} /$ nucleon, as well as in neutron-rich isotopes of elements such as $\mathrm{Ne}$ and $\mathrm{Mg}$ (Leske et al., 1999). Both characteristics had been observed in impulsive events (Mason et al., 1994), but were not expected in gradual events. Figure 2 shows the fluence of $\mathrm{Si}$ (a measure of the event size) plotted versus the $\mathrm{Fe} / \mathrm{O}$ ratio for 76 large SEP events from 1997 to 2003. Note the wide spread in Fe/O. About half of the events have $\mathrm{Fe} / \mathrm{O}$ ratios on either side of the average 5 to $12 \mathrm{MeV} /$ nucleon Fe/O ratio of Reames (1995b), but the distribution is not symmetric - the very largest events tend to be Fe-poor, while the Fe-rich events are on average smaller in intensity.

Although there are exceptions, many Fe-poor events have spectral breaks near $\sim 10 \mathrm{MeV} /$ nucleon, with Fe having a smaller break energy than lighter species such as $\mathrm{O}$ (e.g., Tylka et al., 2000, Cohen et al., 2005). In contrast,

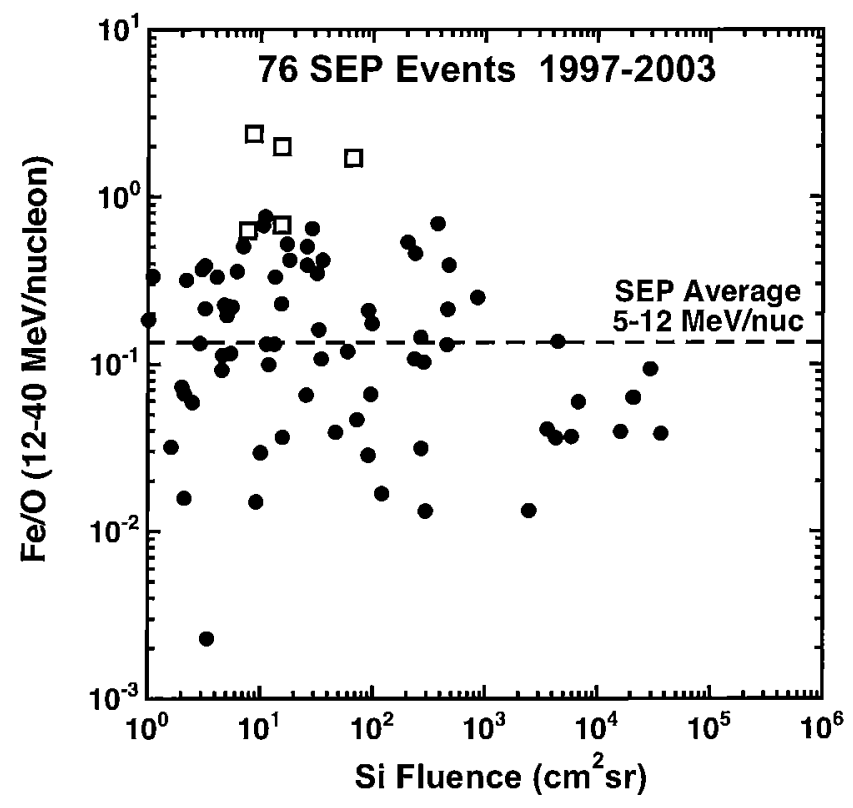

Figure 2. Plot of the fluence of 12 to $40 \mathrm{MeV} /$ nucleon Si versus the $\mathrm{Fe} / \mathrm{O}$ ratio for 76 large SEP events from late 1997 thru 2003. Data are from the SIS instrument on ACE. The average SEP Fe/O ratio of 0.134 determined from 5 to $12 \mathrm{MeV} /$ nucleon measurements is shown for comparison (Reames, 1995b). The diamonds are events identified in the literature as "impulsive". 
many Fe-rich events are power-laws (e.g., Mason et al., 1999a, Cohen et al., 1999). So, while the relative location of the $\mathrm{Fe}$ and $\mathrm{O}$ spectral breaks can explain events below the average SEP line, the large number of Fe-rich events with $\mathrm{Fe} / \mathrm{O} \approx 0.3$ to 1 was not expected, based on studies at lower energy (Reames, 1995b). Many Fe-rich events are also enriched in ${ }^{3} \mathrm{He}$ (Cohen et al., 1999, Cane et al., 2003).

Finally, it was also shown that ionic charge-state patterns in SEP events are more complex than previously believed. Prior to solar cycle $23 \mathrm{Luhn}$ et al. (1984) found that the mean charge states at $\sim 1 \mathrm{MeV} /$ nucleon in twelve gradual events were characteristic of temperatures of $\sim 2$ to $4 \mathrm{MK}$, with some event-to-event variation. Using geomagnetic techniques, Leske et al. (1995) and Tylka et al. (1995) measured similar mean charge states ranging from $\sim 15$ up to several hundred $\mathrm{MeV} /$ nucleon. In contrast, the mean Fe charge-state in 22 impulsive events suggested much higher ( 10 MK) temperatures Klecker et al. (1984).

Once SEP charge-state measurements in the same SEP events became available over a broad energy interval, it was discovered that the mean charge states in most gradual events are energy-dependent, with higher-energy ions more ionized than lower-energy ions (Oetliker et al., 1997, Moebius et al.,
1999, Mazur et al., 1999; Popecki et al., 2003). The mean charge state of $\mathrm{Fe}$ at $\geq 20 \mathrm{MeV} /$ nucleon is often $\approx 20$ (see Figure 3), comparable or greater than that in impulsive events (Mazur et al., 1999, Leske et al., 2001, Labrador et al., 2003; Dietrich and Tylka, 2003).

One suggestion for the increase in the mean charge-state with energy in gradual events is electron stripping. Perhaps high-energy particles traverse more material during acceleration and are stripped of additional electrons (Ruffolo, 1997, Reames, Ng, and Tylka, 1999, Barghouty and Mewaldt, 2000; Kocharov, Kovaltsov, and Torsti, 2001). The amount of electron stripping depends on the product of the density in the acceleration region (n) and the acceleration time ( $\tau$ ). To strip $\mathrm{MeV} /$ nucleon Fe requires $\mathrm{n} \tau \approx 10^{10} \mathrm{~s} / \mathrm{cm}^{3}$ (Kovaltsov et al., 2001). However, there is a correlation between the $\mathrm{Fe} / \mathrm{O}$ ratio and the mean charge state of $\mathrm{Fe}$ (Moebius et al., 2000; Labrador et al., 2003), as shown in Figure 4. It is not obvious why the conditions that lead to stripping in gradual events would favor Fe-rich events.

Following the discovery of energy-dependent charge states in gradual events, it was found that the mean charge states in impulsive events also increase with energy (Moebius et al., 2003, Klecker et al., 2005). Figure 5, includes charge-state
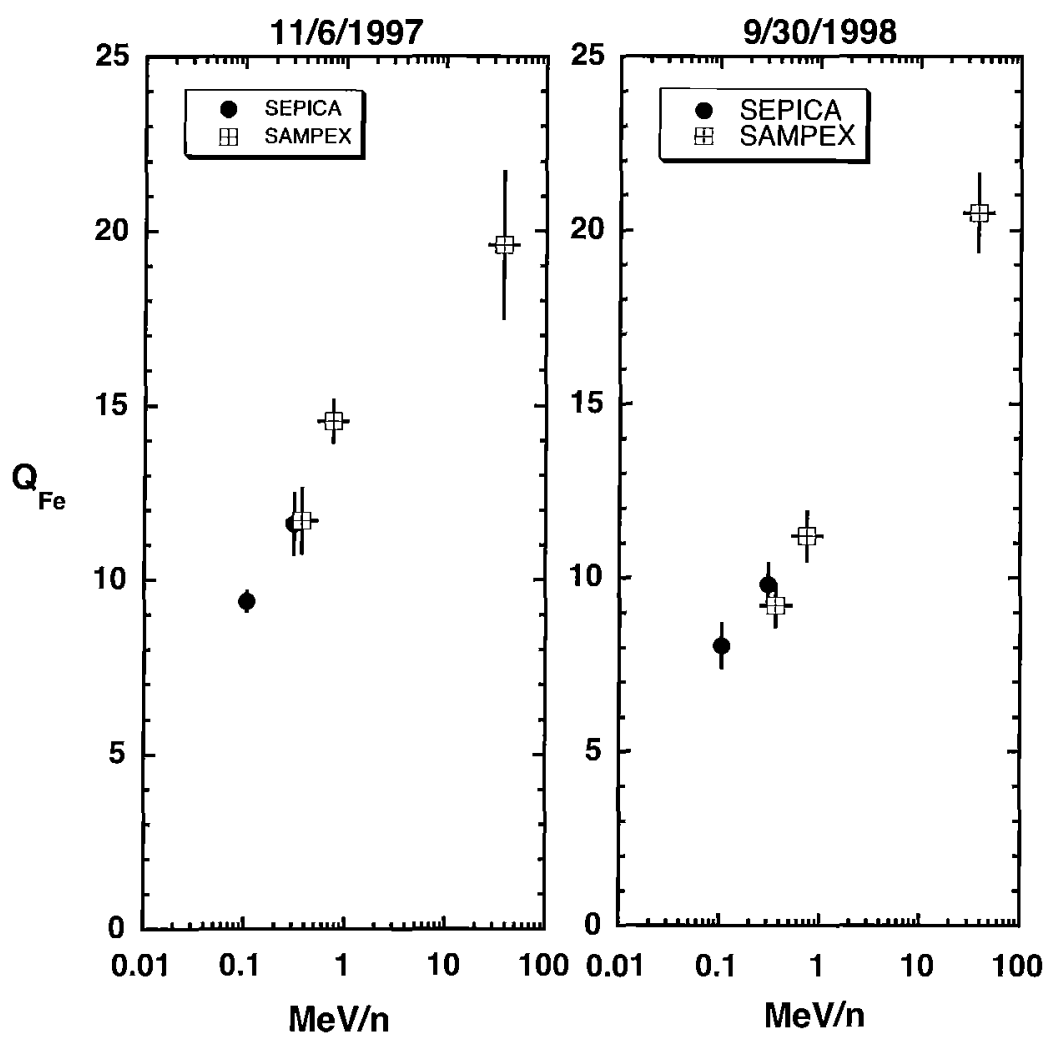

Figure 3. Energy dependence of the Fe charge state measured in two gradual events with data from ACE/SEPICA and from SAMPEX (adapted from Popecki et al., 2003). 


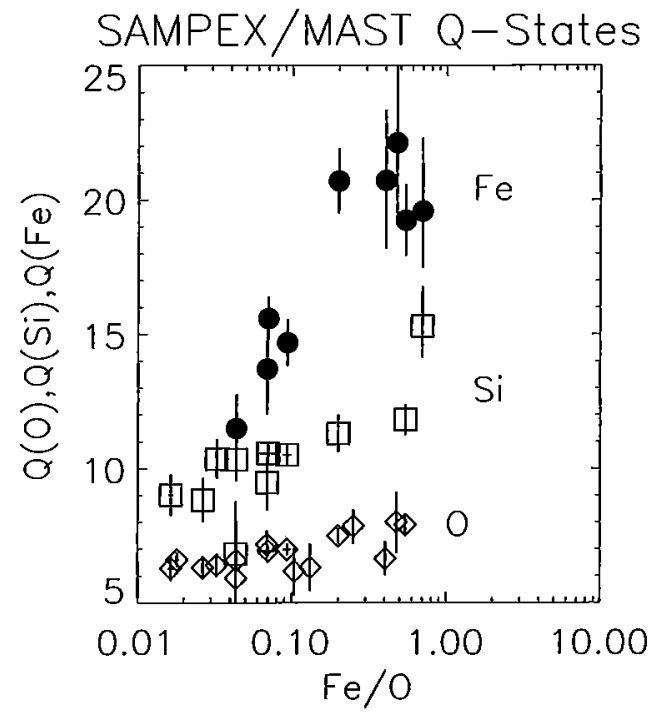

Figure 4. Plot of the mean charge state of $\mathrm{O}, \mathrm{Si}$, and $\mathrm{Fe}$ as measured by SAMPEX versus the $\mathrm{Fe} / \mathrm{O}$ ratio measured by the SIS/ACE instrument (from Labrador et al., 2003). The mean charge state was determined using the geomagnetic method in the energy ranges from 15 to 60,20 to 60 , and 25 to $90 \mathrm{MeV} /$ nucleon for $\mathrm{O}, \mathrm{Si}$, and Fe, respectively.

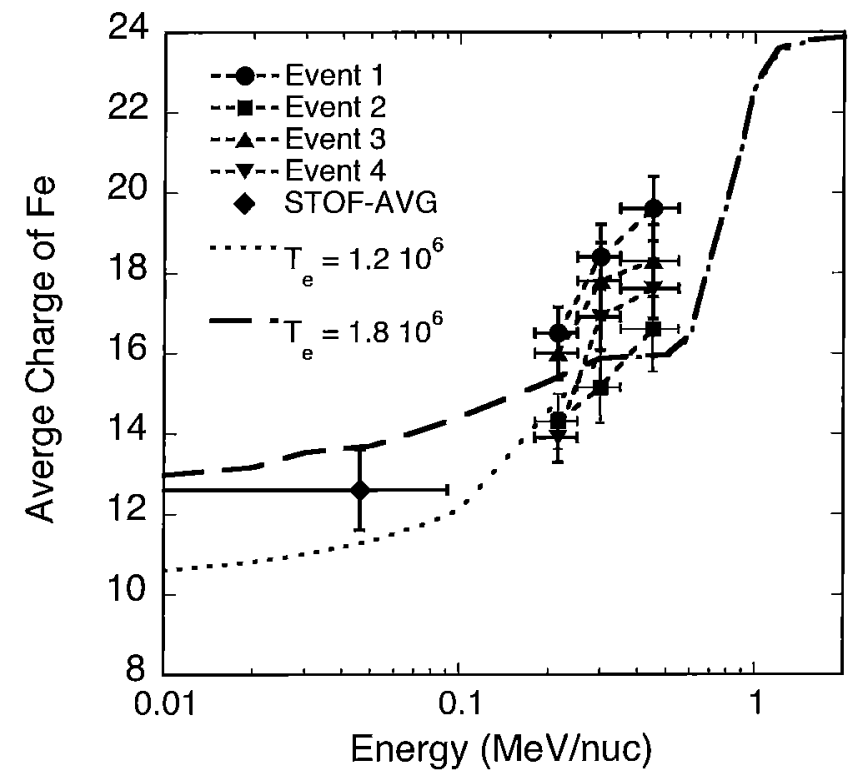

Figure 5. Energy-dependent charge states in impulsive events from Klecker et al. (2005). The curves assume thermal equilibrium for the source material, but allow for electron stripping during acceleration.

measurements from four impulsive events and a theoretical calculation of the equilibrium charge-state distribution. Klecker et al. (2005) concluded from this comparison that in impulsive events the increase in charge state with energy is

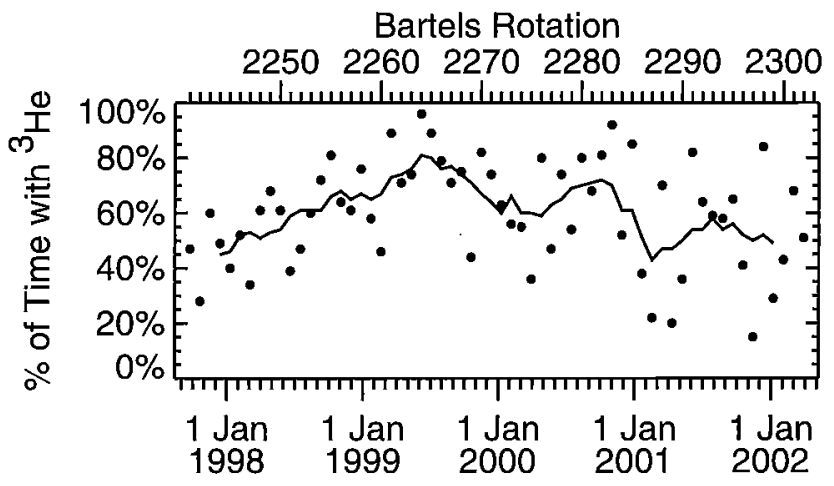

Figure 6. Fraction of time that ${ }^{3} \mathrm{He}$ was present in the interplanetary medium during the years 1998-2001. The solid line is the running mean (based on Wiedenbeck et al., 2002).

due to stripping in the low corona rather than to acceleration of high-temperature material. For further discussion see papers by Popecki (2005) and Kocharov (2005) and Klecker (2005) in this volume.

The combined effect of these new observations has been to blur many of the distinctions between impulsive and gradual events that are based on composition signatures (Mewaldt, 1999; Cohen, 2003, von Rosenvinge and Cane, 2005). The ${ }^{3} \mathrm{He}$ observations, in particular, led to the conclusion that many (and possibly most) gradual events somehow include impulsiveflare material. Because these observations are reminiscent of Cliver's concept of "Hybrid Events" (Cliver, 1996, 2000) that include both flare-accelerated and shock-accelerated particles, this term will be used to denote events that are associated with CME-driven shocks, but have a composition that includes signatures of flare-accelerated particles. The reader is reminded that there are also other properties of SEP events that help to distinguish impulsive and gradual events (Reames, 1995a; von Rosenvinge and Cane, 2005).

\subsection{Suggested Explanations for Hybrid Events}

There have been several suggestions as to how mixtures of flare and gradual material may originate. Mason et al. (1999) suggested that ${ }^{3} \mathrm{He}$ and $\mathrm{Fe}$ enrichments result from remnant interplanetary material from previous impulsive flares that is preferentially accelerated by CME-driven shocks. They showed that ${ }^{3} \mathrm{He}$ is often present in the interplanetary medium (see also Wiedenbeck et al., 2002 and Figure 6).

Tylka et al. (2005) proposed a model that builds on the idea of Mason et al. (1999), but also relies on proposed differences between acceleration at quasi-parallel and quasi-perpendicular shocks (Jokipii, 1982; Giacalone, 2005). Tylka et al., argue that the injection threshold is somewhat greater at quasiperpendicular shocks than at quasi-parallel shocks, and suggest that only suprathermal particles (e.g., $>10 \mathrm{keV} /$ nucleon) 
are accelerated at quasi-perpendicular shocks. This results in a hybrid event if the suprathermal ions are dominated by remnant flare material. In this model the larger, events with normal or Fe-poor composition are due mainly to acceleration at quasi-parallel shocks, in which the shock velocity is great enough to inject solar-wind material with approximately coronal composition.

Cane et al. (2003, 2005) suggested that hybrid events include a mixture of flare and shock-accelerated material, with flare particles contributing at high energies to events with direct connection to the flare site. As support for this suggestion, they cite the fact that the observed $\mathrm{Fe},{ }^{3} \mathrm{He}$, and high-charge-state enrichments occur mainly for events originating at longitudes from W20 to W90, where there is relatively good magnetic connection with the flare site.

Finally, one additional possibility is that the CME-driven shock accelerates flare particles from the associated SEP event in addition to accelerating in situ coronal and interplanetary seed particles (e.g., Mewaldt et al., 2003; Tylka et al., 2005). Li and Zank (2005) have calculated time-intensity profiles and composition patterns that might be expected in such events.

This paper does not attempt to decide between these possibilities, but points out examples of the evidence that should be explained by these models. Any complete model also needs to address other issues including the timing of the associated flare, CME, and particle arrival times, longitudinal and temporal profiles, and observed energy spectra (see Klecker, 2005; Cane et al., 2005; Tylka et al., 2005; Tylka and Lee, 2005; von Rosenvinge and Cane, 2005).

\section{DO GRADUAL SEP EVENTS ACCELERATE SOLAR WIND?}

\subsection{SEP Fractionation According to First Ionization Potential}

Early studies of SEP events discovered that the composition of SEPs is fractionated with respect to photospheric abundances by a selection process that depends on first ionization potential (FIP) or some related atomic parameter (Hovestadt, 1973, Cook et al., 1979, Breneman and Stone, 1985; Meyer et al., 1985; McGuire et al., 1986). Indeed, it was SEP composition studies such as these that first indicated that the coronal composition is fractionated with respect to the photosphere by some ion-neutral selection process (Mewaldt, 1980, Cook, Stone, and Vogt, 1984). A comparison of average SEP and photospheric abundances is shown in the top panel of Figure 7 using average SEP abundances from Reames (1995b) and photospheric abundances from Anders and Grevesse (1989) - note that species with FIP $>\sim 10 \mathrm{eV}$ are depleted with respect to those with FIP $<10 \mathrm{eV}$ by a factor of $\sim 3.3$. Using average SEP abundances from Breneman and Stone (1985) the FIP step is similar, about a factor of 3.6.
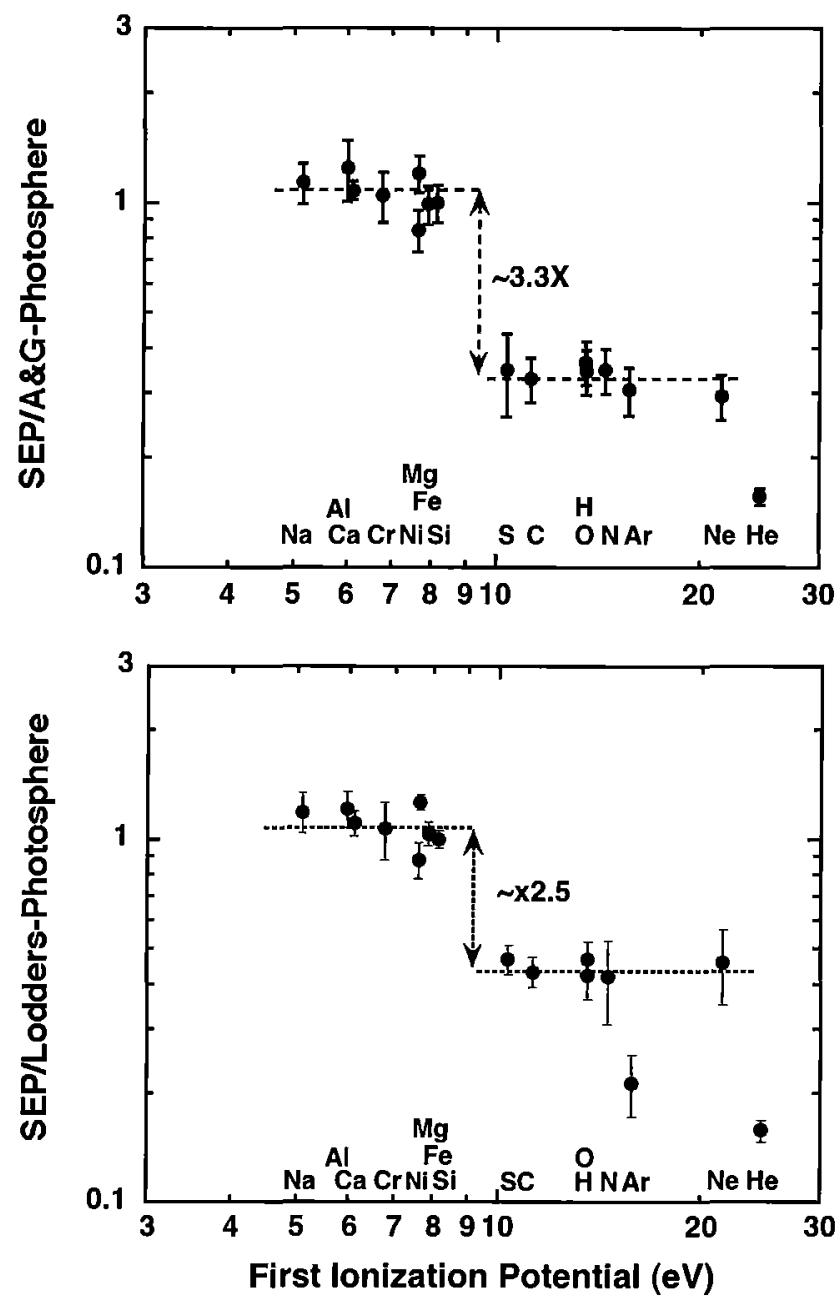

Figure 7. (Top) The ratio of the average SEP abundances with $\sim 5$ to $\sim 12 \mathrm{MeV} /$ nucleon from Reames (1995b) to the photospheric abundances tabulated by Anders and Grevesse (1989) is plotted versus first ionization potential (FIP). Note that elements with FIP $>10 \mathrm{eV}$ are depleted by a factor of $\sim 3.3$ in SEPs. (Bottom) Same as (top), but using photospheric abundances from Lodders (2003). Here the FIP-fractionation step is reduced to 2.5 . In addition, Argon no longer fits the pattern of other high-FIP species.

During recent years there have been significant revisions in the photospheric abundances of $O$ and other volatile elements including $\mathrm{C}$ and $\mathrm{N}$. These changes are reflected in a new table of solar system and photospheric abundances by Lodders (2003). The bottom panel of Figure 7 shows that these revisions reduce the magnitude of the FIP-fractionation step in SEPs from $\sim 3.3$ to $\sim 2.5$ using the average SEP abundances of Reames (1995b).

It has also been suggested that the differences between photospheric abundances and in situ SEP and solar wind abundances are better organized by first ionization time 


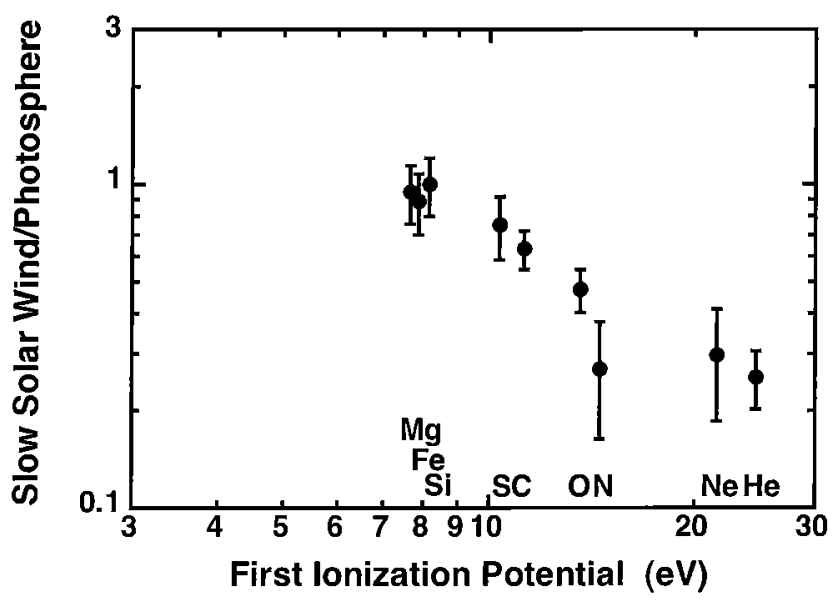

Figure 8. (Top) Ratio of the average slow-speed solar-wind abundances of von Steiger et al. (2000) to the photospheric abundances tabulated by Lodders et al. (2003), plotted vs FIP. A similar dependence is seen for fast solar-wind abundances (see Mewald t et al., $2001 b$ ). Note that CME-driven shocks travel mainly through slow solar wind since there are no active regions in coronal holes.

(FIT) rather than FIP (e.g., Geiss, 1998). However, this possibility does not affect the discussion in the next section.

\subsection{Comparison of Solar-Wind and Solar-Particle Abundances}

Solar-wind abundances also show a FIP fractionation pattern (see Figure 8), but the dependence on FIP appears more like a smooth function than a step function. The differences are seen more clearly in the ratio of average slow solar-wind abundances to SEP abundances (see Figure 9). The ratio of fast solar wind to SEP abundances looks very similar (Mewaldt et al., 2001).

The ratios in Figure 9 are normalized at Si. Note that only 5 of the other 9 ratios are consistent with 1 . Mewaldt et al. (2002) pointed out that several abundance ratios are consistently different in SEPs and solar wind. For example, the $\mathrm{C} / \mathrm{O}$ ratio in SEPs is almost always $\sim 0.4$, while it is $\sim 0.7$ in the solar wind. Similarly, the $\mathrm{S} / \mathrm{Si}$ and the Ne/O ratios also differ, as summarized in Table 1.

One possibility for explaining this situation might be that SEPs have been fractionated as a result of some $\mathrm{Q} / \mathrm{M}$ or mass-dependent acceleration process, as observed, for example by Desai et al. (2003) at interplanetary shocks. However, plotting the ratios in Figure 9 vs. Z, M, or Q/M does not result any simple dependence (see example in Figure 10 and Mewaldt et al., 2001). As a result of these differences, it was concluded that the majority of SEPs are not simply an accelerated sample of bulk solar wind - SEPs must include substantial contributions from other seed populations (Mewaldt et al., 2001, 2002).

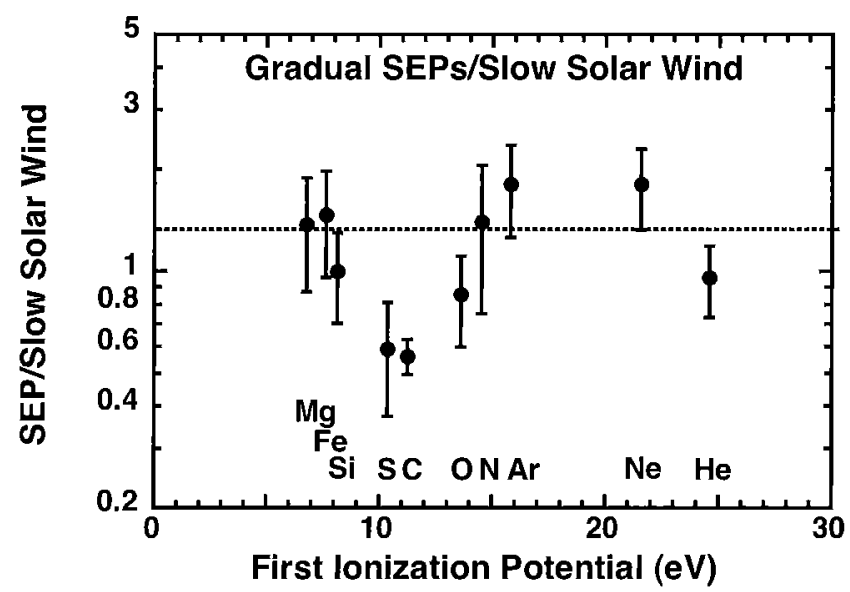

Figure 9. Ratio of the average 5-12 MeV/nucleon SEP abundances from Reames (1995b) to the slow solar-wind abundances of von Steiger et al. (2000), plotted vs FIP. The uncertainties in the average SEP abundances have been added in quadrature with the maximum systematic uncertainties in the SW abundances (statistical uncertainties are negligible for the solar wind measurements).

Table 1. Solar Wind and SEP Abundance Ratios.

\begin{tabular}{lcc}
\hline Ratio & $\begin{array}{c}\text { Slow Solar Wind } \\
\text { (von Steiger et al., 2000) }\end{array}$ & $\begin{array}{c}\text { Solar Particles } \\
\text { (Reames, 1995b) }\end{array}$ \\
\hline $\mathrm{C} / \mathrm{O}$ & $0.67 \pm .08$ & $0.46 \pm .01$ \\
$\mathrm{Ne} / \mathrm{O}$ & $0.10 \pm .03$ & $0.15 \pm .01$ \\
$\mathrm{~S} / \mathrm{Si}$ & $0.33 \pm .16$ & $0.21 \pm .01$ \\
\hline
\end{tabular}

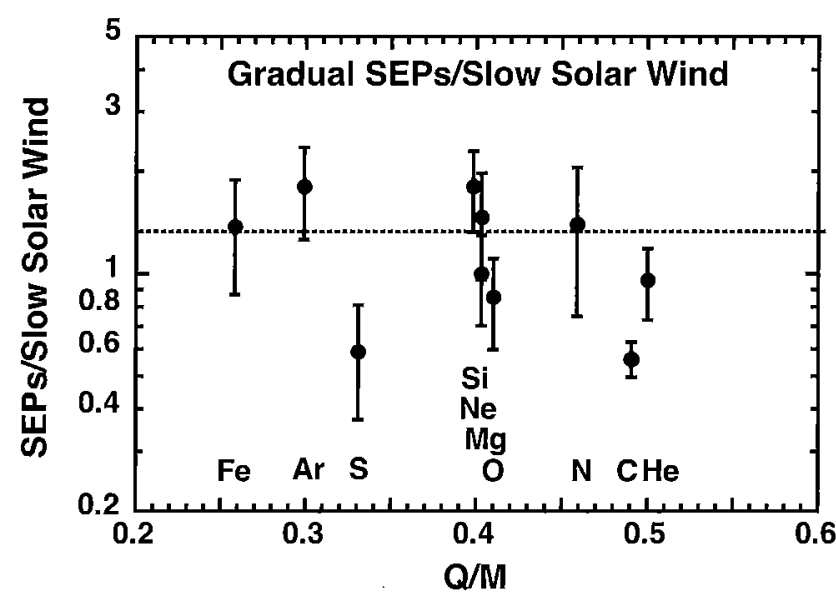

Figure 10. Ratio of average SEP abundances (Reames, 1995b) to slow solar-wind abundances (von Steiger et al., 2000), plotted versus Q/M values from Leske et al. (1995). No simple, monotonic fractionation pattern is seen. 


\section{ARE THERE ENOUGH REMNANT SUPRATHERMALS?}

The interplanetary density of suprathermal seed particles has been measured continuously since late 1997 by the ACE/ULEIS instrument. Figure 11 shows daily-average values of the $\mathrm{Fe}$ intensity versus the $\mathrm{O}$ intensity in the 0.04 to $0.64 \mathrm{MeV} /$ nucleon interval. The composition in this low energy range is surprisingly Fe-rich relative to the average $\mathrm{SEP}$ value of $\mathrm{Fe} / \mathrm{O}=0.134$ at 5 to $12 \mathrm{MeV} /$ nucleon (Reames, $1995 b$ ). It is clear that in addition to a supply of Fe-rich suprathermals from small impulsive events, the material from gradual events is also Fe-rich. Indeed, at all intensity levels, suprathermal SEPs are, on average, Fe-rich. It is therefore not surprising that CME-driven shocks passing through this material would usually accelerate a Fe-rich composition.

The Fe-rich character of low-energy SEP events is demonstrated further in Figure 12, in which the 12 to $40 \mathrm{MeV} /$ nucleon $\mathrm{Fe} / \mathrm{O}$ ratio is plotted against the 0.04 to $0.64 \mathrm{MeV}$ nucleon ratio for 60 large SEP events. Note that for all but two of the 60 events, $\mathrm{Fe} / \mathrm{O}$ is greater than 0.13 at low energy, while at high energy about half the events are Fe-rich and half are Fe-poor. Also identified in Figure 12 are nine hybrid events, identified by the presence of ${ }^{3} \mathrm{He}$ and/or Fe with $\mathrm{Q} \geq 16$ (as well as being Fe-rich at $>12 \mathrm{MeV} /$ nucleon). It appears

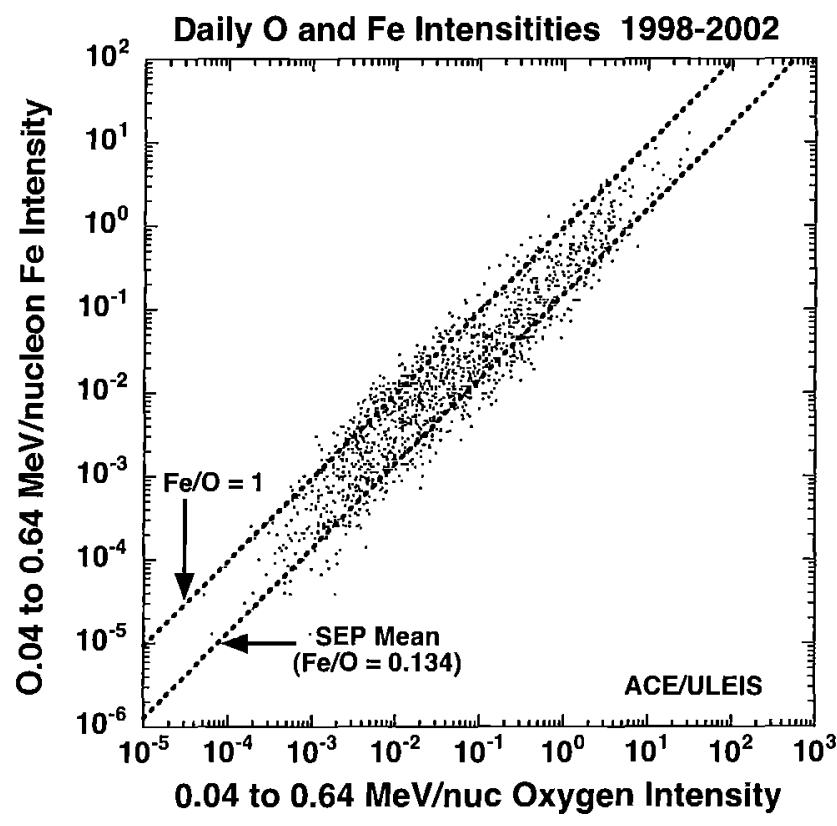

Figure 11. Daily average fluences of 0.04 to $0.64 \mathrm{MeV} /$ nuc Fe are plotted the fluence of 0.04 to $0.64 \mathrm{MeV} / \mathrm{nuc}$ oxygen. There are diagonal lines corresponding the average SEP abundance ratio of 0.134 (Reames, 1998) and to $\mathrm{Fe} / \mathrm{O}=1$. The data are from the ACE/ULEIS instrument.

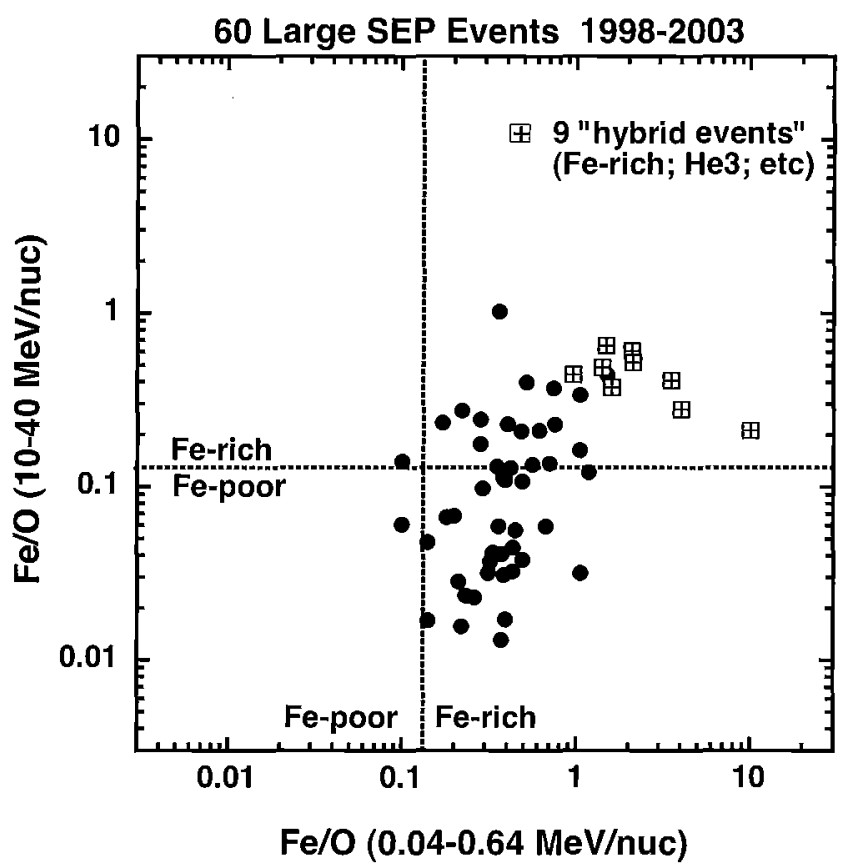

Figure 12. The $\mathrm{Fe} / \mathrm{O}$ ratio at $12-40 \mathrm{MeV} /$ nucleon is plotted against that at 0.04 to $0.64 \mathrm{MeV} /$ nucleon for 60 large gradual SEP events from 1997-2003. The 10 to $40 \mathrm{MeV} /$ nucleon data are from $\mathrm{ACE} / \mathrm{SIS}$ and the 0.04 to $0.64 \mathrm{MeV} /$ nucleon data are from ACE/ULEIS. Nine events indicated by square symbols are examples of hybrid events with $\mathrm{Fe} / \mathrm{O}>0.27$, and either ${ }^{3} \mathrm{He} /{ }^{4} \mathrm{He}>0.001$ or $\mathrm{Q}(\mathrm{Fe}) \geq 16$. The average SEP abundance ratio of 0.134 (Reames, $1995 b)$ is indicated on each axis.

that hybrid events are $\sim 4$ times more Fe-rich at low energy than are typical gradual SEP events.

If the enhanced abundance of ${ }^{3} \mathrm{He}$ and $\mathrm{Fe}$ in hybrid events results from accelerating remnant flare particles from earlier impulsive events (or earlier gradual events), there must be a sufficient density of remnant material available close to the Sun to account for all of the accelerated ions in these events. Mewaldt et al. (2003) surveyed the number density and fluence of suprathermal Fe during 1998-2002 (see Figure 13). The Fe fluence in typical hybrid events (including 4 of the 9 in Figure 12) was $\sim 50$ times greater than the typical quiet-day fluence, and was also much greater than during days when ${ }^{3} \mathrm{He}$-rich events occurred. This implies that typical remnant populations during quiet or moderately active periods are not sufficient to account for the Fe fluence in hybrid eventsthere must be an additional source of Fe. A similar conclusion is reached if we consider the highly-ionized $\left(\mathrm{Q}_{\mathrm{Fe}} \geq 15\right)$ fraction of impulsive SEPs (Figure 5), and try to account for the highly-ionized fraction of hybrid events (Figure 3).

Mewaldt et al. (2003; see also Tylka et al., 2005) suggested that additional $\mathrm{Fe}$ might come from ions accelerated by 


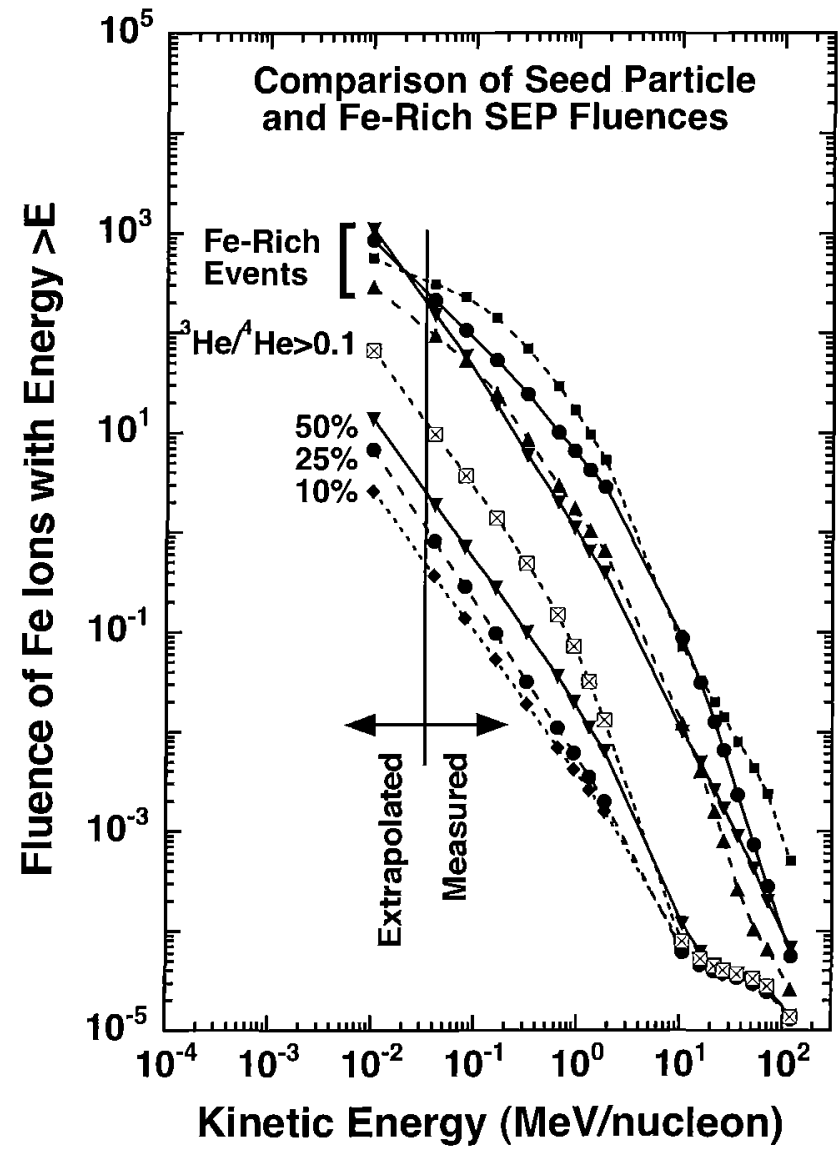

Figure 13. The integrated fluence of $\mathrm{Fe}$ above a given energy is shown for selected time periods and SEP events (based on the study of Mewaldt et al. (2003). The average fluence during quietest $10 \%$, $20 \%$, and $50 \%$ of the days during the period from February 1998 through December 2001 are indicated, as well as the average for days with ${ }^{3} \mathrm{He} /{ }^{4} \mathrm{He}>0.01$. Also shown are Fe fluence spectra from four hybrid events.

the associated flare that are then re-accelerated by the shock. Other possibilities include the injection of even lower-energy ions into the acceleration process, or, the possibility of injection and/or acceleration effects that favor $\mathrm{Fe}$ because of its lower $\mathrm{Q} / \mathrm{M}$ ratio. [However, in interplanetary shocks at $1 \mathrm{AU}$, $\mathrm{Fe}$ is less efficiently accelerated than $\mathrm{O}$ or other light ions (Desai et al., 2003)].

Although one would like to also compare the fluence of ${ }^{3} \mathrm{He}$ in the remnant-flare source and in impulsive and gradual events, ${ }^{3} \mathrm{He}$ is more difficult to measure over a broad energy range under all conditions. The remnant source is more compelling for ${ }^{3} \mathrm{He}$ than for $\mathrm{Fe}$ because impulsive SEP material is the only common source of large ${ }^{3} \mathrm{He} /{ }^{4} \mathrm{He}$ enrichments. The quiet-time $\mathrm{Fe} /{ }^{3} \mathrm{He}$ ratio is $\sim 0.05$ at $\sim 0.1 \mathrm{MeV} /$ nucleon (Mason et al., 1999), while in typical ${ }^{3} \mathrm{He}-\mathrm{rich}$ events $\mathrm{Fe} /{ }^{3} \mathrm{He}$ $\sim 0.1$ (Mason et al., 2002), so quiet-time material has a similar composition to impulsive material. Hybrid events and other gradual events with enriched ${ }^{3} \mathrm{He}$ have $\mathrm{Fe} /{ }^{3} \mathrm{He} \sim 1$ (Mewaldt et al., 2003), so they have relatively more Fe than impulsive events. In addition, there is apparently a ceiling on the ${ }^{3} \mathrm{He}$ fluence in SEP events (Ho et al., 2005). Thus, if the remnant source is responsible for the ${ }^{3} \mathrm{He}$ in gradual events, the remnant contribution to $\mathrm{Fe}$ would again appear to fall short by a factor of $\sim 10$ to $\sim 20$.

Although it would be revealing if we could measure suprathermal ions close to the Sun to compare with the characteristics of SEP events, this is presently difficult because suprathermals take $\sim 1$ day to get to 1 AU. However, we can measure the suprathermal population the day before an event (reflecting conditions near the Sun $\sim 2$ days before the event). This comparison relies on the fact that suprathermal ion intensities change rather slowly because these ions are not very mobile. Surprisingly, the $>0.04 \mathrm{MeV}$ /nucleon $\mathrm{Fe} / \mathrm{O}$ ratio on days before hybrid events was not any more Fe-rich than for more normal or Fe-poor events. However, during hybrid events the $>0.04 \mathrm{MeV} /$ nucleon $\mathrm{Fe} / \mathrm{O}$ ratio was much more Fe-rich than normal (see Figure 12).

The other interesting finding is that most hybrid events do not occur during quiet periods. For 6 of 8 hybrid events the $>0.04 \mathrm{MeV} /$ nucleon $\mathrm{Fe}$ intensity on the previous day was among the top $25 \%$ most-active days; for only one event was the previous day among the 50\% least-active days (see Figure 13). For other gradual SEP events only 13 of 33 occurred after the $25 \%$ most-active days. So, it appears that another solution to the source problem is to accelerate remnant material from earlier, large, gradual events, which happens quite naturally when several SEP events occur within a few days. While the approach used here does not sample conditions close to the Sun at the onset of the event (the previous day is only a rather crude proxy), it does indicate that hybrid events occur mainly during periods of high activity rather than during quiet periods. In this case the most plentiful source of suprathermal ions may be material from previous gradual events, which are inherently Fe-rich on average (see Figures 11 and 12).

\section{OTHER SOURCES OF SEED PARTICLES}

Other possible sources of seed particles should also be considered. It is known that suprathermal particles are injected more easily into the shock acceleration process than thermal particles (Gloeckler et al., 1994, Desai et al., 2003). Interstellar (IS) singly-charged pickup ions are accelerated at the termination shock, but there does not appear to be efficient acceleration of the bulk solar wind. (Cummings, Stone and Steenberg, 2002). At interplanetary shocks near 1 AU IS pickup $\mathrm{He}$ is efficiently accelerated, but IS He is not available close to the Sun (Kucharek et al., 2003). It also appears that 
inner-source pickup ions do not make a significant contribution to gradual SEP events.

ICME material is another possible source of seed particles. There is evidence that larger SEP events often result when a fast CME follows a slower CME (Gopalswamy et al., 2002). Whether this is a result of "CME interactions" (Gopalswamy et al., 2002) or "preconditioning" of the medium by the first ICME (Kahler et al., 2003) is unclear, but in this situation the shock from the 2nd CME may pass through freshly ejected CME material, which often has highly-ionized Fe and other anomalous abundances (e.g., Lepri et al., 2004). Lepri et al., suggest that the highly-ionized material is produced by electron beams from the associated flare or injected directly from the flare site.

Mason (2001) has described the pool of suprathermal ions in the inner heliosphere. In addition to remnant flare particles, there are remnant particles from previous gradual and CIR events, and also CME material. In addition, there are ubiquitous suprathermal tails on the solar wind (Geiss et al., 1995; Gloeckler et al., 2000) with roughly $\mathrm{E}^{-2}$ energy spectra starting at several times the solar-wind velocity (see Figure 1). The origin of these tails is not known, but they apparently result from interplanetary acceleration process(es) because there are tails on interstellar pickup He as well as on all common solar-wind species. It is not yet clear if these tails are sufficiently intense to be a viable source of seed particles for the largest SEP events, and it is also not known if their composition can explain the composition differences described in Section 2.2 and Figure 9. Further investigation of the properties of suprathermal solar-wind tails could address several important questions about the source of SEP seed material.

\section{SUMMARY}

The wealth of new SEP data from solar maximum 23, supplemented by the first comprehensive solar-wind composition measurements, and improved solar observations from SOHO, TRACE, and RHESSI has led to significant evolution of our picture of SEP events. The new data covering multiple species over broad energy ranges has shown that CME-driven shocks accelerate more than the ambient solar wind, which had previously been the presumed source material for shockassociated SEP events. By realizing that the compositional signatures of SEPs are not due solely to acceleration and transport mechanisms, SEP researchers have changed the paradigm for probing the physical processes operating in these events.

To account for the observed enhancement of ${ }^{3} \mathrm{He}$ in gradual events, material from impulsive SEP events must somehow be incorporated. This has generated fresh ideas for models that include mechanisms such as direct contributions of flare-accelerated particles, or the shock acceleration of either remnant flare material, or flare material from the associated flare. New SEP ionization-state measurements give evidence for stripping of SEPs, which implies acceleration much closer to the Sun. CME-driven shocks sometimes accelerate material more closely resembling that of the corona, but the FIP-fractionation patterns observed in SEP events differ in several key respects from those in the solar wind. Although theoretical studies indicate that fast CMEdriven shocks can, in principle, accelerate the bulk solar wind, studies at $1 \mathrm{AU}$ suggest that it is predominantly suprathermal ions that are accelerated. This revolutionizes our interpretation of SEP abundances, and makes clear the need to thoroughly understand the suprathermal populations, and the mechanisms that lead to their preferential acceleration in shock-associated events.

The composition of SEP events varies considerably with energy. In the energy range below $1 \mathrm{MeV} /$ nucleon, it is surprising that essentially all SEP events are Fe rich, with typical $\mathrm{Fe} / \mathrm{O}$ ratios of $\sim 0.5$, compared to typical solar wind values of $\sim 0.2$ and average SEP values of $\sim 0.13$ at 5 to $12 \mathrm{MeV} /$ nucleon. Because of the higher intensities at lower energy, the bulk SEP composition must also be Fe-rich. This suggests that either (1) the seed material is Fe-rich (possibly derived from flare material), or (2) there are rigidity-dependent injection/acceleration effects operating near the Sun that favor ions with larger $\mathrm{M} / \mathrm{Q}$ ratios such as $\mathrm{Fe}$.

At higher energies, $>10 \mathrm{MeV} /$ nucleon, the composition is more variable, with $\mathrm{Fe} / \mathrm{O}$ ratios varying by $\sim 2$ orders of magnitude from event to event. Much of this variation is due to spectral effects - SEP events commonly exhibit Q/M-dependent spectral breaks, in which the break energy is located at higher energy/nucleon for lighter species with higher Q/M ratios. However, in many cases, the Fe-rich character observed at lower energies continues to higher energies. There is a marked tendency for hybrid events to be more Fe-rich than average below $1 \mathrm{MeV} /$ nucleon. In addition, hybrid events tend to have power-law spectra that preserve the low-energy composition rather than distorting it.

Whatever source of seed material is considered, it is important to establish how it is accelerated to produce the observed fluences in the largest SEP events. Simple considerations show that this is not easy, thus illustrating challenges ahead in the modeling these events. As an example of the issues, the fluence $>10 \mathrm{keV} /$ nucleon Fe ions on typical quiet days is $\sim 10$ times less than the $>10 \mathrm{keV} /$ nucleon fluence in typical hybrid events, and $\sim 100$ times less than the fluence during the largest SEP events. Therefore, to supply the needed material in large events the seed particles must either be (1) derived from energies $<10 \mathrm{keV} /$ nucleon (e.g., heated solar wind or CME material), or (2) derived from remnant material from previous gradual and impulsive events, or (3) derived directly or indirectly from flare particles originating in the associated 
flare. It is important that these processes be modeled to determine whether the candidate seed populations can be injected and accelerated to yield the observed spectra and composition.

New missions, both planned or proposed, can give critical insights into these issues. STEREO, supplemented by in situ and imaging data from near Earth, will investigate longitudinal variations in the composition and energy spectra and identify the relative contributions of shock and flare-accelerated particles. Missions that venture closer to the Sun (Inner Heliospheric Sentinels, Solar Orbiter, and Solar Probe) will provide measurements of the available seed populations, plasma, and interplanetary conditions closer to the Sun, where most of the acceleration takes place. In addition, global models of particle acceleration by shocks are now exploring more realistic computations of shock acceleration scenarios. Taken together, these advances in observation and theory/modeling should make possible quantitative tests of the entire process of particle acceleration and propagation in the inner heliosphere.

Acknowledgments. This work was supported by NASA under grants NNG04GB55G, NNG04088G, and NAG5-12929. We appreciate discussions with Berndt Klecker, Eberhard Moebius, and Edward Stone, and figures provided by Berndt Klecker, Allan Labrador, Mark Popecki, and Mark Wiedenbeck. We thank the local organizing committee for a very informative and enjoyable conference.

\section{REFERENCES}

Anders, E., and N. Grevesse, Abundances of the elements: meteoritic and solar, Geochim. Cosmochim. Acta, 53, 197-214, 1989.

Barghouty, A.F., and R.A. Mewaldt, Simulation of charge-equilibrium and acceleration of solar energetic ions, in Acceleration and Transport of Energetic Particles Observed in the Heliosphere, AIP Conf, Proc. 528, 71-78, 2000.

Breneman, H.H., and E.C. Stone, Solar coronal and photospheric abundances from solar energetic particle measurements, Astrophys. I. Lett. 299, L57-L61, 1985.

Cane, H.V., T.T. von Rosenvinge, C.M.S. Cohen, and R.A. Mewaldt, Two components in major solar particle events, Geophys. Res. Lett., 30, SEP 5-1, doi: 10.1029/ 2002GL016580, 2003.

Cane, H.V., R.A. Mewaldt, C.M.S. Cohen, and T.T. von Rosenvinge, The role of CMEs and shocks in determining SEP abundances, submitted to J. Geophys. Res., 2005.

Cliver, E.W., Solar flare ganma ray emission and energetic particles in space, in High Energy Solar Physics, R. Ramaty el al., Eds, AIP Conf. Proc. 374, 45-60, 1996.

Cliver, E.W., Solar flare photons and energetic particles in space, in Acceleration and transport of energetic particles in the heliosphere, A.I.P. Conf. Proc. 528, 21-31, 2000.

Cohen, C.M.S., Solar energetic particle acceleration and interplanetary transport, in Frontiers of Cosmic Ray Science, T. Kajita et al., Editors, Universal Academy press, Tokyo, pp. 113-134, 2004.

Cohen, C.M.S. et al., New observations of heavy-ion-rich solar particle events from ACE, Geophys. Res. Lett., 26, 2697-2700, 1999.

Cohen, C.M.S., et al., Heavy ion abundances and spectra from the large SEP events of October-November 2003, J. Geophys. Res., 110, doi:101029/2005JA0I 1004, 2005.

Cook, W.R., E.C. Stone, and R.E. Vogt, Elemental composition of solar energetic particles, Astrophys. J., 279, 827-838, 1984.

Cummings, A.C., E.C. Stone, and C.D. Steenberg, Composition of anomalous cosmic rays and other heliospheric ions, Astrophys. J, 578, 194-210, 2002.

Desai, M.I. ef al., Evidence for a suprathermal seed population of heavy ions accelerated by interplanetary shocks near I AU, Astrophys. J., 588, 1149-1162, 2003.

Dietrich, W.F., and A.J. Tylka, Time to maximum studies and inferred ionic charge sates in the solar energetic particle events of 14 and 15 April, 2001, $28^{\text {th }}$ Internat. Cosmic Ray Conf., 6, 3291-3294, 2003.
Ellison, D.C., and R. Ramaty, Shock acceleration of electrons and ions in solar flares, Astrophys. J., 298, 400-408, 1985.

Geiss, J., G. Gloeckler, L.A. Fisk, and R. von Steiger, Pickup ions in the heliosphere and their origin, J. Geophys. Res., 1000, 23373-23377, 1995.

Geiss, J., Constraints on the FIP mechanisms from solar wind abundance data, Space Sci. Rev., 85, 241-252, 1998.

Giacalone, J., Astrophys. J., 628, L37-L40, 2005.

Gloeckler, G., L.A. Fisk, T.H. Zurbuchen, and N.A. Schwadron, in Acceleration and Transport of Energetic Particles in the Heliosphere, AIP Conf. Proc. 528, 229-233, 2000.

Gloeckler; G., et al., Acceleration of interstellar pickup ions in the disturbed solar wind observed on Ulysses, J. Geophys. Res., 99(A9), 17637-17643, 1994.

Gopalswamy, N., et al., Interacting coronal mass ejections and solar energetic particles, Astrophys. J. Lett., 572, L103-L106, 2002.

Ho, G., E.C. Roelof, and G.M. Mason, The upper limit on ${ }^{3} \mathrm{He}$ fluence in solar energetic particle events, Astrophys. J., 621, L141-L144, 2005.

Hovestadt, D. (1973), "Nuclear composition of solar cosmic rays", in Solar Wind Three C.T. Russell, Ed., IGPP/UCLA, Los Angeles, pp. 2-25, 1973.

Jokipii, J.R., Particle drift, diffusion, and acceleration at shocks, Astrophys. J., 255 716-720, 1982.

Klecker, B., et al., Direct determination of the ionic charge distribution of helium and iron in He-3-rich solar energetic particle events, Astrophys. J., 281, 458-462, 1984.

Klecker, B.E., et al., Observation of energy-dependent ionic charge states in impulsive solar energetic particle events, Adu. Space Res., in press, 2005.

Kocharov, L.G., Modeling the energy-dependent charge states of solar energetic particles, this volume, 2005.

Kocharov, L., and J. Torsti, Hybrid solar energetic particle events observed on SOHO, Solar Phys., 207, 149-157, 2002.

Kovaltsov, G.A., A.F. Barghouty, L. Kocharov, V.M. Ostryakov, and J. Torsti, Chargeequilibrium of Fe ions accelerated in a hot plasma, Astron. Astrophys., 375, 1075-1081, 2001.

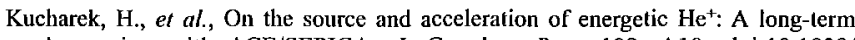
observation with ACE/SEPICA, J. Geophys. Res., 108, A10, doi:10.1029/ 2003JA009938, 2003.

Lepri, S. T., and T. H. Zurbuchen, Iron charge state distributions as an indicator of hot ICMEs: Possible sources and temporal and spatial variations during solar maximum, J. Geophys. Res., 109(Al), doi:10.1029/2003JA009954, 2004.

Leske, R.A., et al., Unusual isotopic composition of solar energetic particles observed in the November 6, 1997 event, Geophys. Res. Lett., 26, 153-156, 1999.

Leske, R.A., et al., The ionic charge state composition at high energies in large solar energetic particle events in solar cycle 23, in Solar and Galactic Composition, AIP Conf. Proc. 598, 171-176, 2001.

Leske, R.A., et al., Measurements of the ionic charge states of solar energetic particles using the geomagnetic field, Astrophys. J., 452, L149-L152, 1995.

$\mathrm{Li}, \mathrm{G}$, and G.P. Zank, Mixed particle acceleration at CME-driven shocks and flares, Geophys. Res. Letl., 32, L02101, doi:10.1029/2004GL021250, 2005.

Li, G., G.P. Zank, and W.K.M. Rice, Acceleration and transport of heavy ions at coronal mass ejection-driven shocks, submitted to J. Geophys. Res., 2005.

Lodders, K., Solar system abundances and condensation temperatures of the elements, Astrophys. J., 591, 1220-1247, 2003.

Luhn, A., et al., Ionic Charge States of $\mathrm{N}, \mathrm{Ne}, \mathrm{Mg}, \mathrm{Si}$, and $\mathrm{S}$ in Solar Energetic Particle Events, Adv. Space Res., 4(2), 161-166, 1984.

Mason, G.M., Heliospheric lessons for galactic cosmic-ray acceleration, Space Sci. Rev., 99, 119-133, 2001.

Mason, G.M., J.E. Mazur, and D.C. Hamilton, Heavy-ion isotopic anomalies in ${ }^{3} \mathrm{He}$-rich solar particle events, Astrophys. J., 425, 843-848, 1994.

Mason, G.M., et al., Particle acceleration and sources in the November 1997 solar energetic particle events, Geophys. Res. Lett., 26, 141-144, 1999a.

Mason, G.M., J.E. Mazur, and J.R. Dwyer, ${ }^{3} \mathrm{He}$ enhancements in large solar energetic particle events, Astrophys. J. Lett., 525, L133-L136, 1999b.

Mason, G.M., et al., Spectral properties of $\mathrm{He}$ and heavy ions in ${ }^{3} \mathrm{He}$-rich solar flares, Astrophys. J., 574, 1039-1058, 2002.

Mazur, J.E., G.M. Mason, B. Klecker, and R.E. McGuire, The energy spectra of solar flare hydrogen, helium, oxygen, and iron: evidence for stochastic acceleration, Astrophys. J. 401, 398-410, 1992.

Mazur, J.E. et al., Charge states of solar energetic particles using the geomagnetic cutoff technique: SAMPEX measurements in the 6 November 1997 solar particle event, Geophys. Res. Lett., 226, 173-176, 1999.

McGuire. R.E., T.T. von Rosenvinge, and F.B. McDonald, The composition of solar energetic particles, Astrophys. J., 302, 938-961, 1986.

Mewaldt, R.A., Spacecraft measurements of the elemental and isotopic composition of solar energetic particles, in Proceedings of the Conference on the Ancient Sun, eds, R.O. Pepin, et al., Pergamon, New York, pp. 81-101, 1980.

Mewaldt, R.A., New views of solar energetic particles from the Advanced Composition Explorer, Proceedings of the 26th International Cosmic Ray Conference, Invired, Rapportew; and Highlight Papers, AIP Conf. Proc. 516, 265-270, 2000.

Mewaldt, R.A., et al., Long-term fluences of energetic particles in the heliosphere, in Solar and Galactic Composition, AIP Conf. Proc. 598, 165-170, 2001. 
Mewaldt, R.A., el al., Impulsive flare material: A seed population for large solar particle events? 281h Internat. Cosmic Ray Conf., 6, 3329-3332, 2003.

Mewaldt, R.A., et al., Solar energetic particle spectral breaks, in Physics of Collisionless Shocks, AIP Conf. Proc., 227-232, 2005.

Mewaldt $e$ al., Fractionation of solar energetic particles and solar wind according to first ionization potential, Adv Space Res., 30(1), 79-84, 2002.

Mewaldt, R.A., et al., Are solar energetic particles an accelerated sample of solar wind? R.A. Mewaldt, et al., Proc. 27th Internal. Cosmic Ray Conf., 8, 3132-3135, 2001.

Meyer, J.P., The baseline composition of solar energetic particles, Astrophys. J. Supp., $57,151-171,1985$.

Moebius, E., et al., Strong energy dependence of ionic charge states in impulsive solar events, 281h Internat. Cosmic Ray Conf., 6, 3273-3276, 2003.

Moebius, E., et al., Energy dependence of the ionic charge state charge state distribution during the November 7-9 solar energetic particle event observed by ACE SEPICA, Geophys. Res. Lett., 226, 145-148, 1999.

Moebius, E., et al., Survey of ionic charge states of solar energetic particle events during the first year of ACE, in Acceleration and Transport of Energetic Particles Observed in the Heliosphere, AIP Conf, Proc. 528, 131-134, 2000.

Oetliker, M., et al., The ionic charge of solar energetic particles with energies of $0.3-70 \mathrm{MeV}$ per nucleon, Astrophys. J., 477, 495, 1997.

Popecki, M.A., Observations of energy-dependent charge states in solar energetic particles, this volume, 2005.

Popecki, M.A. et al., Proc. 28th Internat. Cosmic Ray Conference, 6, 3283-3286, 2003.

Reames, D.V., Solar energetic particles: A paradigm shift, Rev. of Geophys., 33, 585-589, 1995a.

Reames, D.V., Coronal abundances determined from energetic particles, Adv. Space Res., 15(7), 41-51, 1995b.

Reames, D.V, C.K. Ng, and A.J. Tylka, Energy-dependent ionization states of shockaccelerated particles in the solar corona, Geophys. Res. Letf., 26, 3585-3588, 1999.

Ruffolo, D., Charge states of solar cosmic rays and constraints on acceleration times and coronal transport, Astrophys. J. Lett., 481, L119-L122, 1997.
Torsti, J., J. Laivola, and L. Kocharov, Common overabundance of ${ }^{3} \mathrm{He}$ in high-energy solar particles, Astron. Astrophys., 408, L1-L4, 2003.

Tylka. A.J., et al., The mean ionic charge state of solar energetic Fe ions above $200 \mathrm{MeV}$ per nucleon, Astrophys. J. Lett., 444, L109-L113, 1995.

Tylka, A.J., D.V. Reames, and C.K. Ng, Observations of systematic temporal evolution in elemental composition during gradual solar energetic particle events, Geophys. Res. Lett., 26, 2141-2144, 1999.

Tylka, A.J., P.R. Boberg, R.E. McGuire, C.K. Ng, and D.V. Reames, Temporal evolution in the spectra of gradual solar energetic particle events, in Acceleration and Transport of Energetic Particles Observed in the Heliosphere, A.I.P. Conf. Proc. 528, $147-152,2000$.

Tylka, A.J., et al., Shock geometry, seed populations, and the origin of variable elemental composition at high energies in large gradual solar particle events, Astrophys. J., 625, 474-495, 2005.

Tylka, A.J., and M.A. Lee, this volume, 2005.

von Rosenvinge, T.T., and H.V. Cane, Overview of solar energetic particles, this volume, 2005 .

von Steiger, R., et al., Composition of quasi-stationary solar wind flows from the Ulysses Solar-Wind Ion Composition Spectrometer, J. Geophys. Res., 105(A12), 27217-27238, 2000.

Wiedenbeck, M.E., et al., Enhanced abundances of ${ }^{3} \mathrm{He}$ in large solar energetic particle events, in Acceleration and transport of energetic particles observed in the heliosphere, AIP. Conf. Proc. 528, 107-110, 2000.

Wiedenbeck, M.E., et al., How common is energetic ${ }^{3} \mathrm{He}$ in the inner heliosphere? in Solar Wind 10, AIP Conf. Proc. 679, 652-655, 2003.

C.M.S. Cohen, 220-47 Downs Laboratory, Caltech, Pasadena, CA 91125

G.M. Mason, Johns Hopkins University, Applied Physics Laboratory, Johns Hopkins Rd, Laurel, MD 20723-6099

R.A. Mewaldt, 220-47 Downs Laboratory, Caltech, Pasadena, CA 91125 